\title{
Effects of topical chondrocyte-derived extracellular matrix treatment on corneal wound healing, following an alkali burn injury
}

\author{
JAE-WOOK YANG ${ }^{1,2}$, SOUNG-MIN LEE ${ }^{2-4}$, KYEONG-HEE OH ${ }^{2-4}$, \\ SAE-GWANG PARK ${ }^{2-4}$, II-WHAN CHOI ${ }^{2-4}$ and SU-KIL SEO ${ }^{2-4}$ \\ ${ }^{1}$ Department of Ophthalmology, Busan Paik Hospital; ${ }^{2}$ Ocular Neovascular Research Center, College of Medicine; \\ ${ }^{3}$ Departments of Microbiology and ${ }^{4}$ Immunology, College of Medicine, \\ Inje University, Busan 614-735, Republic of Korea
}

Received January 27, 2014; Accepted August 29, 2014

DOI: $10.3892 / \mathrm{mmr} .2014 .2722$

\begin{abstract}
Numerous treatments have been used in the management of corneal chemical burns; however, no optimal treatment for corneal chemical burns currently exists. The present study investigated the effects of topical chondrocyte-derived extracellular matrix (CD-ECM) treatment on corneal wound healing, using an alkali burn mouse model. Topical treatment with CD-ECM was shown to reduce corneal opacity following an alkali burn. A histological examination observed the presence of regenerated epithelial cells and a small number of inflammatory cells in the corneas of CD-ECM-treated mice. The majority of the inflammatory cells present in the corneas of the phosphate-buffered saline (PBS)-treated mice were neutrophils that expressed matrix metalloproteinase (MMP)-9. The amount of neutrophils was significantly reduced in the corneas of the CD-ECM-treated mice. Furthermore, the expression levels of interleukin (IL)-8 were significantly reduced in the CD-ECM treatment group, but not in the mice that received the PBS treatment. The results of the present study indicate that CD-ECM treatment may accelerate wound healing in a model of alkali burn-induced corneal injury. The therapeutic mechanism may be associated with accelerated reepithelialization and reduced recruitment of MMP-9-expressing neutrophils, through inhibiting the production of IL-8.
\end{abstract}

\section{Introduction}

Chemical burns can cause severe injury to corneal tissues. These burns often result in a marked infiltration of

Correspondence to: Dr Su-Kil Seo, Department of Microbiology, College of Medicine, Inje University, Bokji-ro 75, Busanjin-gu, Busan 614-735, Republic of Korea

E-mail: sseo@inje.ac.kr

Key words: cornea, chemical burn, extracellular matrix inflammatory cells, extensive formation of scar tissue, recurrent erosion of the epithelium, ulceration, stromal edema and neovascularization (1-3). These processes often lead to a significant loss of corneal transparency. Numerous treatments have been developed to manage corneal chemical burns $(4,5)$; however, there is currently no optimal treatment for corneal chemical burns.

Corneal epithelial cells undergo a process of self-renewal, in order to replace surface layer cells and repair corneal surface wounds $(6,7)$. Therefore, the fast recovery of epithelial cells following an acute corneal injury is important for wound healing $(8,9)$. Impaired reepithelialization, following corneal injury, leads to the recruitment of inflammatory cells that destruct corneal tissues. Inflammatory chemokines, cytokines and matrix metalloproteinases (MMPs) are essential factors in these inflammatory responses (10).

The components of the extracellular matrix (ECM) include: Glycosaminoglycans, proteoglycans, glycoproteins, and collagens. These components have previously been shown to promote tissue renewal and healing, following injury $(11,12)$. During injury repair and tissue engineering, the ECM induces the surrounding cells to repair the wounded tissue, instead of forming scar tissue. The ECM also prevents the triggering of immune responses that are associated with inflammation. Collagen has previously been introduced as a wound-healing agent used for the treatment of burns and skin ulcerations $(13,14)$. Treatment with collagen leads to the formation of a collagen corneal shield, which acts as an ocular surface bandage (15). Collagen shields have been investigated using both animal and human subjects, and have been shown to promote corneal epithelial and stromal healing $(16,17)$. Hyaluronic acid (HA), a glycosaminoglycan (GAG), has demonstrated anti-inflammatory properties in enhancing wound repair $(18,19)$. Therefore, in response to the findings of previous studies, the clinical application of ECM materials has been studied in the regeneration of injured tissues.

Previous reports have shown the potential use of chondrocyte-derived extracellular matrix (CD-ECM), isolated from porcine chondrocytes, as a biomaterial scaffold. The composition of the CD-ECM scaffold consists of: $73 \%$ collagen type I, $16 \%$ GAGs, and 11\% water (20). The CD-ECM scaffold 
supports chondrogenic differentiation of mesenchymal stem cells (MSCs) and helps maintain the phenotype in vivo $(21,22)$. The aims of the present study were to determine the wound-healing effects of CD-ECM on corneal injury and to elucidate the cellular mechanism underlying these effects.

\section{Materials and methods}

Animals. The female outbred imprinting control region mice were purchased from Charles River Laboratories (Tokyo, Japan) between the ages of six and seven weeks. The mice were handled according to the guidelines in the Association for Research in Vision and Opthalmology Statement for the Use of Animals in Opthalmic and Vision Research. The animal protocols were approved by the Institutional Animal Care and Use Committee of Inje University (Busan, South Korea) (ethical approval no. 2012-034).

Preparation of CD-ECM solution. The CD-ECM was provided by RegenRrime Co., Ltd. (Suwon, South Korea), and was prepared as described by previous methods (20). Briefly, primary chondrocytes were isolated from porcine knee joints and were initially expanded in a monolayer culture for three weeks, and further cultured in a 3-dimensional (3-D) pellet for another three weeks. The ECM scaffold was produced from the 3-D pellet, following removal of the chondrocytes. The scaffold was treated with $200 \mathrm{U} / \mathrm{ml}$ DNase I and washed completely with phosphate-buffered saline (PBS). Finally, the scaffold was dried and converted to a powder. To prepare the solution for topical application, the CD-ECM was dissolved at various concentrations in PBS. $1 \%$ CD-ECM in PBS was the highest concentration to be used for the topical treatment. The $1 \% \mathrm{CD}$-ECM was vortexed vigorously for $5 \mathrm{~min}$ and centrifuged at $300 \mathrm{xg}$ for $5 \mathrm{~min}$. The supernatant was collected and stored at $4^{\circ} \mathrm{C}$ until further use.

Alkali burn. The mice were anesthetized with an intraperitoneal injection of $5 \mathrm{mg} / \mathrm{kg}$ rompun (Bayer, Monheim, Germany) and $40 \mathrm{mg} / \mathrm{kg}$ zoletil (Virbac, Carros Cedex, France). To induce the alkali burns, $3 \mu 1$ of $1.0 \mathrm{~N} \mathrm{NaOH}$ was instilled onto the entire surface of the right cornea for $18 \mathrm{sec}$. The ocular surface was then rinsed with $10 \mathrm{ml}$ PBS. After one day, the mice were randomly separated into two treatment groups, and CD-ECM $(10 \mu 1$ of $1 \%, \mathrm{n}=10)$ or PBS $(10 \mu \mathrm{l}, \mathrm{n}=10)$ was topically applied to the corneas three times daily, between days 1-7. The eyes of the mice were observed daily using slip lamp microscopy (SL-D7; Topcon Medical System Inc., Oakland NJ, USA) under a dim lighting condition. For this procedure, live mice were anesthetized and handled with care each time opacity was observed in their cornea.

Clinical observation. The corneal opacity was determined based on the following parameters, as previously described (8): 0 , no opacity; $1,<1 / 3$ of the corneal surface was clouded; $2,<2 / 3$ of the corneal surface was clouded; $3,>2 / 3$ of the corneal surface was clouded; 4 , almost all of the corneal surface was clouded.

Histological examination. Four days following the alkali burn, a subset of the mice from each group $(n=5)$ were sacrificed.
The eyeballs of these mice were fixed in $10 \%$ buffered formalin and embedded in paraffin. The paraffin sections were cut into $5 \mu \mathrm{m}$ and stained with hematoxylin and eosin (H\&E). The corneal morphology was assessed using a NanoZoomer 2.0 RS (Hamamatsu, Shizuoka, Japan).

Immunohistochemical detection. Four days following the alkali burn, a subset of the mice from each group $(n=5)$ were sacrificed by carbon dioxide $\left(\mathrm{CO}_{2}\right)$ inhalation. The eyeballs were harvested and embedded in paraffin for immunohistochemical staining, to detect the expression levels of MMP-9. The sections were deparaffinized and boiled in antigen retrieval solution (Vector Laboratories Inc., Burlingame, CA, USA), at $121^{\circ} \mathrm{C}$ for $5 \mathrm{~min}$. The endogenous peroxidase activity was blocked by $3 \%$ hydrogen peroxide treatment for $10 \mathrm{~min}$. Nonspecific staining was blocked by treatment with CAS-Block $^{\mathrm{TM}}$ (Invitrogen Life Technologies, Carlsbad, CA. USA) for $10 \mathrm{~min}$. The sections were then incubated with the primary monoclonal rat anti-mouse MMP-9 antibody (R\&D Systems Inc., Minneapolis, MN, USA) diluted in CAS-Block ${ }^{\mathrm{TM}}$ (1:50), overnight at $4^{\circ} \mathrm{C}$. Following three washes with PBS for $5 \mathrm{~min}$, the sections were incubated with horseradish peroxidase-conjugated secondary anti-rat Immunoglobulin G antibody (Santa Cruz Biotechnology Inc., Dallas, TX, USA), for 1 hour at room temperature, followed by a further wash with PBS. The reaction product was developed using 3, $3 \mathrm{~N}$-diaminobenzidine tetrahydrochloride for $5 \mathrm{~min}$. The sections were then counter-stained with Mayer hematoxylin. The corneal morphology was assessed using the NanoZoomer $2.0 \mathrm{RS}$.

Reverse-transcription (RT) and quantitative (q) polymerase chain reaction $(P C R)$. The corneal samples were obtained on days four and seven, following the alkali burn. Total RNA was extracted from the corneal samples (pooling five corneas from each group) using TRIzol ${ }^{\circledR}$ reagent (Invitrogen Life Technologies). The RNA was reverse-transcribed into cDNA using a PCR cDNA Synthesis kit (Clontech Laboratories Inc., Mountain View, CA, USA). The RT-PCR was performed using sense/antisense primers. The qPCR (45 cycles) was performed using SYBR-green Supermix (Bio-Rad Laboratories, Hercules, CA, USA) and run on an iCycler System (Bio-Rad Laboratories), according to the manufacturer's instructions. The PCR and qPCR primer sequences are shown in Table I. The Ct values were calculated as the ratio of the SYBR fluorescence. Each sample was normalized by the $\mathrm{Ct}$ value of GAPDH.

Statistical analyses. A student's t-test was used for the statistical analysis of the clinical scores and qPCR. A P $<0.05$ was considered to indicate a statistically significant difference.

\section{Results}

Effects of CD-ECM on alkali burn-induced corneal injury. The effects of topical CD-ECM treatment were investigated on corneal injuries, following an alkali burn. In the PBS treatment group the corneal opacity was observed from day 2 following the alkali burn, and markedly increased in severity in a time-dependent manner. Conversely, the corneal opacity 
Table I. Primer sets used for polymerase chain reaction (PCR) and quantitative PCR.

\begin{tabular}{|c|c|c|c|c|}
\hline Gene & Sequence $\left(5^{\prime}-3^{\prime}\right)$ & Size (bp) & Annealing temp $\left({ }^{\circ} \mathrm{C}\right)$ & PCR cycle \\
\hline GAPDH & $\begin{array}{l}\mathrm{F}: \text { TTCACCACCATGGAGAAGGC } \\
\mathrm{R}: \text { GGCATGGACTGTGGTCATGA }\end{array}$ & 244 & 58 & 23 \\
\hline IL-8/KC & $\begin{array}{l}\mathrm{F}: \text { CAGCCACCCGCTCGCTTCTC } \\
\mathrm{R}: \text { TCAAGGCAAGCCTCGCGACCAT }\end{array}$ & 223 & 58 & 30 \\
\hline MMP-9 & $\begin{array}{l}\mathrm{F}: \text { CTGGACAGCCAGACACTAAA } \\
\mathrm{R}: \text { CTCGCGGCAAGTCTTCAGAG }\end{array}$ & 145 & 58 & 30 \\
\hline VEGF & $\begin{array}{l}\mathrm{F}: \text { TTACTGCTGTACCTCCACC } \\
\mathrm{R}: \text { ACAGGACGGCTTGAAGATG }\end{array}$ & 189 & 58 & 30 \\
\hline IL-1 $\beta$ & $\begin{array}{l}\mathrm{F}: \text { CTCGTGCTGTCGGACCCATAT } \\
\mathrm{R}: \text { TTGAAGACAAACCGCTTTTCCA }\end{array}$ & 253 & 58 & 30 \\
\hline IL-6 & $\begin{array}{l}\mathrm{F}: \text { TGGGAAATCGTGGAAATGAG } \\
\mathrm{R}: \text { GAAGGACTCTGGCTTTGTCTT }\end{array}$ & 249 & 58 & 30 \\
\hline MMP-2 & $\begin{array}{l}\mathrm{F}: \text { CCCCGATGCTGATACTGA } \\
\mathrm{R}: \text { CTGTCCGCCAAATAAACC }\end{array}$ & 152 & 58 & 30 \\
\hline TNF- $\alpha$ & $\begin{array}{l}\mathrm{F}: \text { CCACACCGTCAGCCGATTTG } \\
\mathrm{R}: \text { CACCCATTCCCTTCACAGAG }\end{array}$ & 255 & 58 & 30 \\
\hline CXCL-1 & $\begin{array}{l}\mathrm{F}: \text { GTGTCCCCAAGTAACGGAGA } \\
\mathrm{R}: \text { TGCACTTCTTTTCGCACAAC }\end{array}$ & 317 & 58 & 30 \\
\hline CXCL-2 & $\begin{array}{l}\mathrm{F} \text { : AGTTTGCCTTGACCCTGAAGCC } \\
\mathrm{R}: \text { TGGGTGGGATGTAGCTAGTTCC }\end{array}$ & 468 & 58 & 30 \\
\hline
\end{tabular}

F, forward; R, reverse; bp, base pairs; PCR, polymerase chain reaction.

of the group treated with CD-ECM was markedly lower, as compared with the PBS treatment group, and it did not increase in a time-dependent manner (Fig. 1). These data indicate that CD-ECM treatment may inhibit corneal injury, following an alkali burn.

CD-ECM treatment accelerates corneal wound healing. The effects of CD-ECM were evaluated using histologic examination of the corneas. Prior to the topical treatment with CD-ECM or PBS, the corneal epithelium was completely destructed by an alkali burn at day 1 (Fig. 2A), as compared with the normal cornea (Fig. 2B). Consistent with the previous clinical scores, the corneas of the PBS treatment group were markedly thicker, had higher levels of inflammatory cell infiltration and their epithelium remained destructed (Fig. 2C); however, recovered epithelial cells and a smaller number of inflammatory cells were present in the corneas of the CD-ECM treatment group (Fig. 2D). These results indicate that topical treatment with CD-ECM may enhance the repair of corneal epithelial cells and inhibit the infiltration of inflammatory cells.

$C D$-ECM treatment inhibits the expression levels of MMP-9. To identify the inflammatory factors associated with the decreased corneal opacity following CD-ECM treatment the corneas were collected at days 4 and 7, following the alkali burn, and RT followed by qPCR was performed. There were no significant differences in the expression levels of vascular endothelial growth factor (VEGF), IL-1 $\beta$, tumor necrosis factor (TNF)- $\alpha$, or MMP-2 between the PBS and CD-ECM treatment groups
A

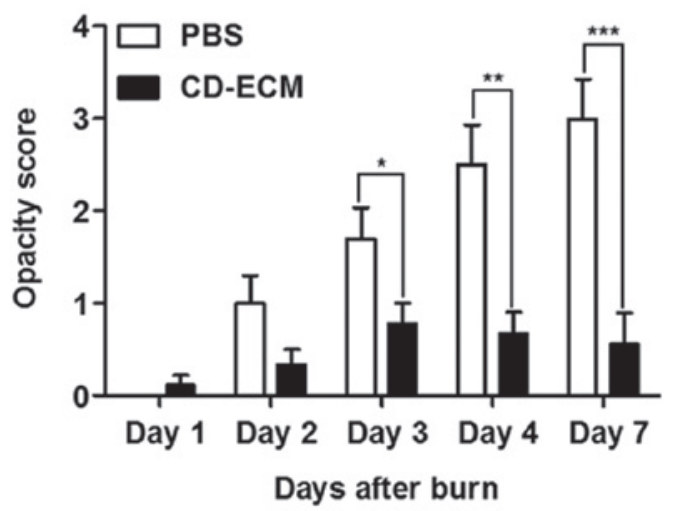

B

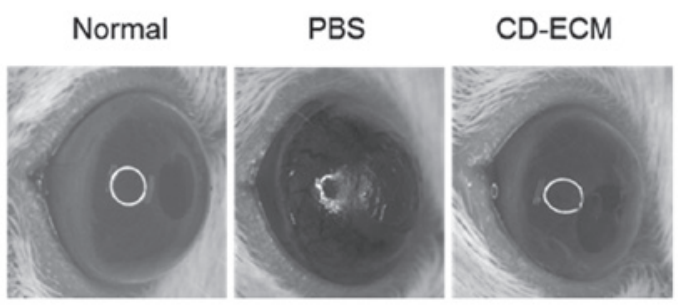

Figure 1. Effects of chondrocyte-dervied extracelullar matrix (CD-ECM) on alkali burn-induced corneal injury. (A) Clinical score of corneal alkali burn in the CD-ECM- and phosphate-buffered saline (PBS)-treated mice. The corneas of the mice were treated with $1.0 \mathrm{~N} \mathrm{NaOH}$ solution. CD-ECM and PBS were applied topically to the corneas three times daily between days 1 and 7 . The corneal opacity was scored as described previously. The clinical score data are presented as the means \pm standard deviation. Concordant results were obtained from three independent experiments. ${ }^{*} \mathrm{P}<0.05,{ }^{* *} \mathrm{P}<0.01$, and ${ }^{* * * *} \mathrm{P}<0.001$ for the PBS versus the CD-ECM group. (B) Representative images of the cornea at day 7 following an alkali burn (magnification, x80). 
A

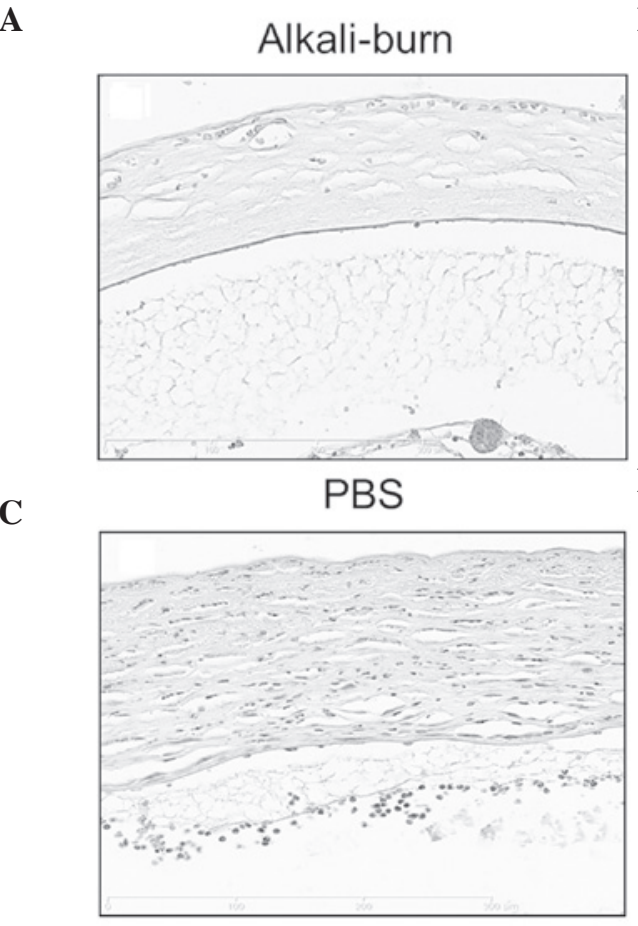

B

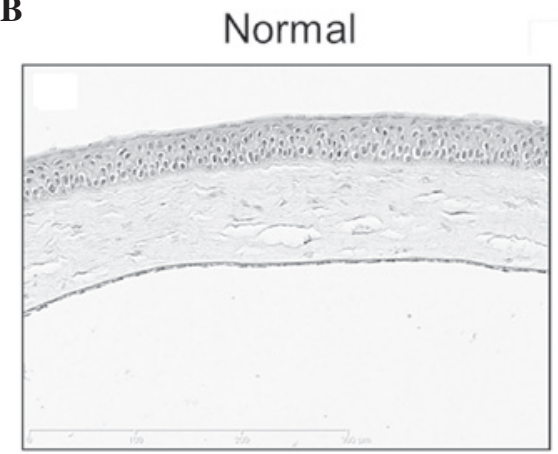

D

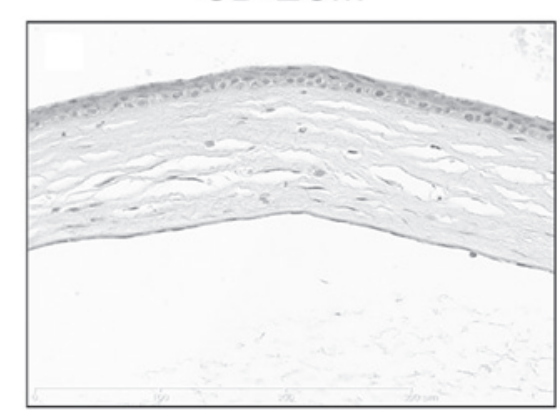

Figure 2. Chondrocyte-dervied extracelullar matrix (CD-ECM) treatment accelerates corneal model wound healing, as determined by histological examination of the corneas. (A) The corneas of the mice were treated with $1.0 \mathrm{~N} \mathrm{NaOH}$ solution (alkali-burn). After one day, the corneas were collected and stained with hematoxylin and eosin and compared with (B) the normal corneas. (C) Phosphate-buffered saline (PBS) or (D) CD-ECM was applied topically to the burned corneas three times daily between days 1 and 4 . The corneas were collected at day 4 . Concordant results were obtained in three independent experiments. Magnification, x200.

A

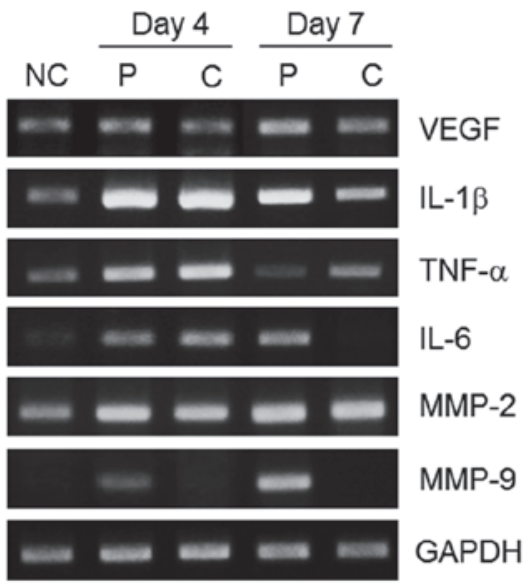

B

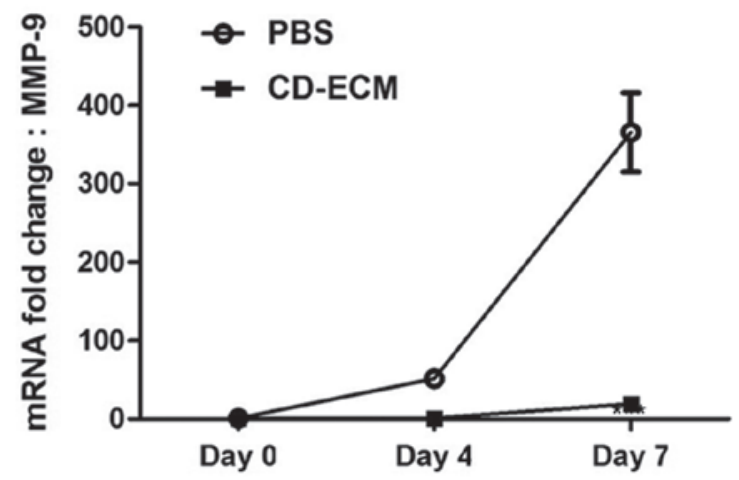

Figure 3. Chondrocyte-dervied extracelullar matrix (CD-ECM) treatment inhibits the expression levels of matrix metalloproteinase (MMP)-9. (A) Expression profiles of pro-inflammatory factors in CD-ECM- and phosphate-buffered saline (PBS)-treated corneas. The corneas of the mice were treated with $1.0 \mathrm{~N}$ NaOH solution. After one day, CD-ECM was applied topically on the corneas three times daily. The corneas $(n=5)$ were collected on days 4 and 7 . Total RNA was extracted from the corneas and reverse transcribed into cDNA. A polymerase chain reaction (PCR) was performed using the specific primers shown in Table I, and the products were analyzed by agarose gel electrophoresis. The results are representative of three experiments. (B) Quantitative PCR analysis of MMP-9 expression levels. The results are expressed as the mean ratio of MMP-9/GAPDH \pm standard deviation. The data represent two combined experiments. ${ }^{* * *} \mathrm{P}<0.001$, the PBS versus the CD-ECM group. VEGF, vascular endothelial growth factor; IL, interleukin; TNF, tumor necrosis factor; MMP, matrix metalloproteinase.NC, normal corneas; P, PBS; C, CD-ECM.

at either day 4 or 7 (Fig. 3A). The expression levels of IL-6 levels were similar at day 4; however, they were reduced in the CD-ECM treatment group at day 7, as compared with the PBS treatment group. There was a marked difference in the expression levels of MMP-9 between the PBS and CD-ECM treatment groups. At day 4, the expression levels of MMP-9 were induced in the corneas of the PBS-treated mice, and the levels were significantly lower in the corneas of the CD-ECM-treated mice. Furthermore, the expression levels of MMP-9 were markedly increased in the PBS-treated corneas at day 7, whereas the expression levels in the CD-ECM-treated corneas at day 7 were similar to those at day 4 (Fig. 3B). These results suggest that CD-ECM treatment may inhibit the upregulation of MMP-9 expression in corneas, following an alkali burn. 
A

B

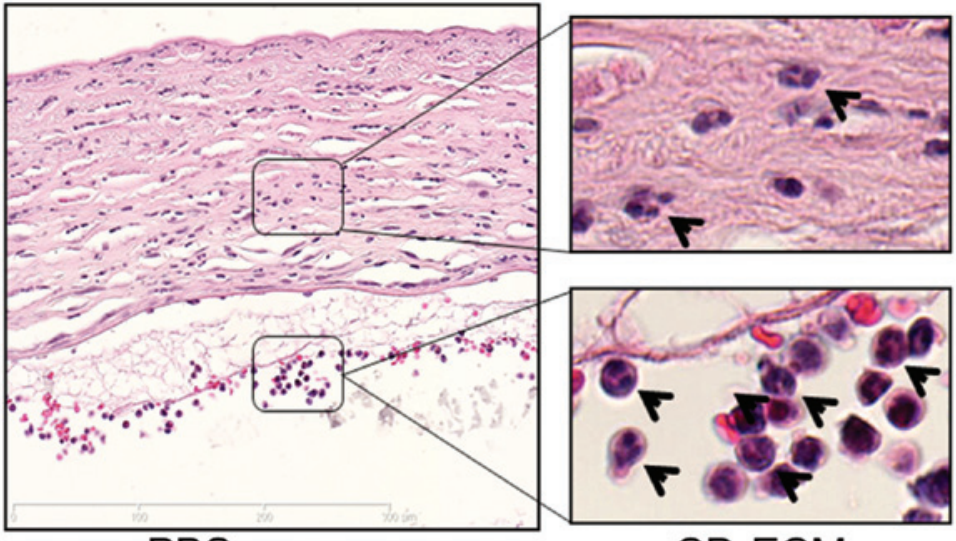

PBS

CD-ECM
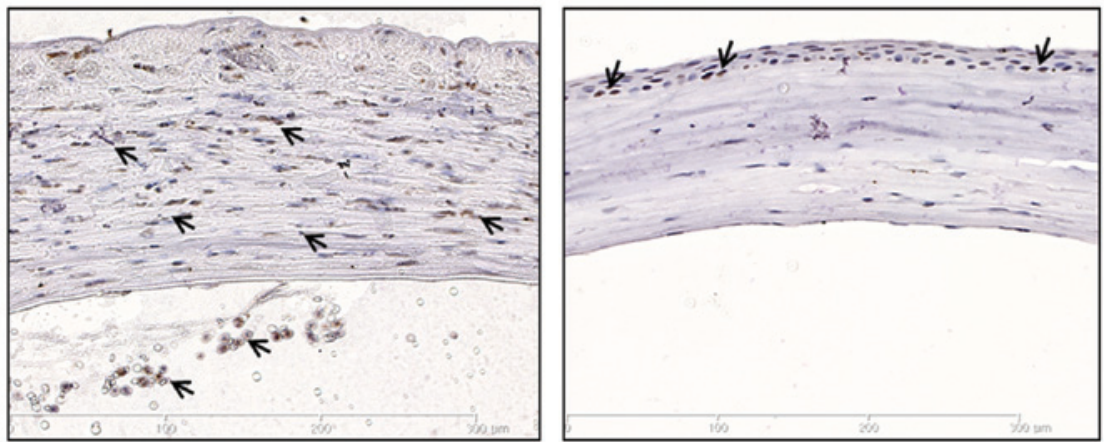

Figure 4. Chondrocyte-derived extracelullar matrix (CD-ECM) treatment inhibits the recruitment of matrix metalloproteinase (MMP)-9 expressing neutrophils. (A) Histologic examination was used to detect neutrophils, as represented by the arrows. The corneas were collected at day 4 following an alkali burn and stained with hematoxylin and eosin. The results are representative of three experiments. (B) A section of a cornea stained to detect MMP-9 expressing cells, as represented by the arrows. The corneas were collected from CD-ECM and phosphate-buffered saline (PBS) treatment groups at day 4 following an alkali burn. Magnification, x200.

A

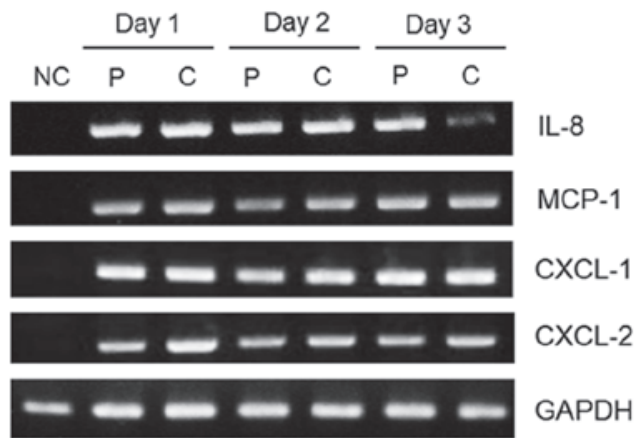

B

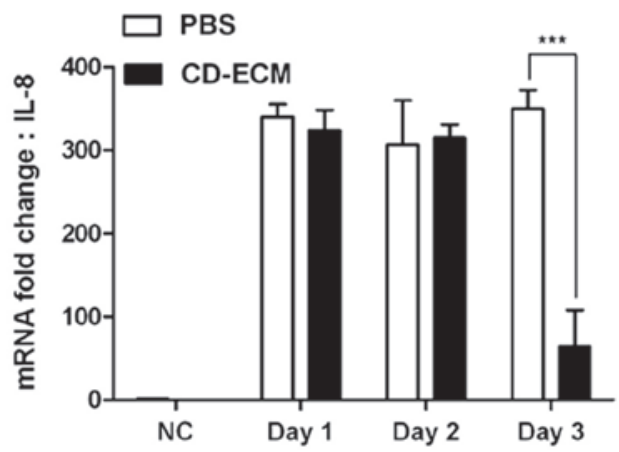

Figure 5. Chondrocyte-dervied extracelullar matrix (CD-ECM) treatment inhibits the production of interleukin (IL)-8. (A) Expression profiles of chemokines in CD-ECM- and phosphate-buffered saline (PBS)-treated corneas. The corneas of the mice were treated with $1.0 \mathrm{~N} \mathrm{NaOH}$ solution. After one day, CD-ECM was applied topically to the corneas three times daily. The corneas $(n=5)$ were collected at the indicated time points. Total RNA was extracted from the corneas and reverse transcribed into cDNA. A polymerase chain reaction (PCR) was performed using the specific primers shown in Table 1, and the products were analyzed by agarose gel electrophoresis. The results are representative of three experiments. NC, normal corneas; P, PBS; C, CD-ECM. (B) Semi-quantitative PCR analysis of IL-8 expression levels. The results are expressed as the mean ratio of IL-8/GAPD \pm standard deviation. The data represent two combined experiments. ${ }^{* * *} \mathrm{P}<0.001$ for PBS versus CD-ECM. MCP, monocyte chemoattractant protein; CXCL, chemokine ligand.

CD-ECM treatment inhibits the recruitment of neutrophils, a major source of MMP-9 expression. CD-ECM treatment was shown to inhibit the upregulation of MMP-9 expression levels following an alkali burn; therefore, the present study aimed to determine the cellular source of MMP-9 expression, using immunohistochemical staining. The H\&E staining demonstrated that the infiltrating cells were predominantly neutrophils, which were identified by the "horse shoe" shape of their nuclei (Fig. 4A). The expresssion of MMP-9 was observed in the neutrophils from the corneas of the PBS-treated mice; however, MMP-9 expression was observed in several epithelial cells in the corneas of the CD-ECM-treated mice (Fig. 4B). These may be healing 
epithelial cells, as these cells have previously been identified as being a cellular source of MMP-9 expression in wound healing conditions (23). There were few MMP-9-expressing neutrophils in the CD-ECM treatment group. These data suggest that the suppressed expression of MMP-9 may be due to the inhibitory effects of CD-ECM treatment on neutrophil recruitment.

CD-ECM treatment inhibits IL-8 production. Chemokines have been strongly implicated as crucial mediators of neutrophil influx. Therefore the present study investigated the expression levels of chemokines associated with neutrophil recruitment, including IL-8, chemokine ligand (CXCL)-1, CXCL-2, and monocyte chemoattractant protein-1, using qPCR. All of these chemokines were induced by alkali burn and were maintained in the corneas of the PBS treatment group. In the corneas from the CD-ECM treatment group, the expression levels of IL- 8 were reduced by day 3 (Fig. 5A). The decrease in IL- 8 expression levels, as determined by qPCR, at day 3 in the CD-ECM treatment group were significantly reduced, as compared with the PBS treatment group (Fig. 5B). However, no differences were observed in the expression levels of the other chemokines between the two groups. These data indicate that the decrease in IL-8 expression levels may be responsible for the inhibition of the influx of neutrophils into the corneas of CD-ECM-treated mice following an alkali burn.

\section{Discussion}

Chemical burn of the cornea is a common ocular injury. Slow epithelialization, persistent ulceration, corneal perforation and angiogenesis are common complications associated with the acute phase of corneal chemical burn. Clinical management during the acute phase of corneal chemical burn is important; however, there remains a lack of appropriate management. Novel ways to immediately and appropriately treat the acute phase of corneal chemical burns are required (24).

Due to the ability of the components of the ECM to promote wound healing, the clinical application of ECM materials have been studied in the regeneration of injured tissues (25). Previous studies have shown that CD-ECM may serve as a biomaterial scaffold. The CD-ECM scaffold supports chondrogenic differentiation of MSCs and helps maintain the phenotype of these cells in vivo $(21,22)$. This phenomenon is responsible for the major composition of CD-ECM involved in collagen type II and HA. Therefore, it was hypothesized in the present study that CD-ECM may be used to heal corneal chemical burns. Soluble CD-ECM was prepared from its sponge form (20) and its wound healing effects were evaluated by the topical administration of CD-ECM against corneal alkali burn. Prior to treatment with CD-ECM or PBS, the corneas were observed for neovascularization and destruction of the epithelium, following an alkali burn. The corneal opacity was significantly increased in the PBS treatment group; however, treatment with CD-ECM markedly reduced corneal opacity. Using histological analysis on corneas isolated on day 4, regenerating epithelial cells and few infiltrated inflammatory cells were observed in the CD-ECM-treated mice. These results suggest that topical CD-ECM treatment facilitated the repair of corneal epithelial cells, following injury by alkali burn and may inhibit the further infiltration of inflammatory cells.
Impaired reepithelialization is associated with heightened inflammation and insufficient stromal remodeling, resulting in the loss of transparency of the corneal tissues (10). The production of proinflammatory factors, including IL-1 $\beta$, TNF- $\alpha$, IL-6, and VEGF, has been shown to occur quickly in alkali-burned corneas (26). These cytokines are released from the injured corneal epithelial cells and lead to the induction of the angiogenic response and keratocyte apoptosis (27). In the present study CD-ECM treatment did not significantly affect the expression levels of these factors, following an alkali burn. Based on these data, it may be hypothesized that CD-ECM promotes the repair of injured corneal epithelial cells and does not reduce the expression levels of IL-1 $\beta$, TNF- $\alpha$, IL-6, and VEGF produced by the epithelial cells. Notably, CD-ECM treatment strongly prevented the upregulation of the expression levels of MMP-9. MMP-9 is predominantly expressed by neutrophils (28) and aids the infiltration of these cells into non-lymphoid tissues and enhances tissue destruction (29). Neutrophils were demonstrated to be the predominant cell type that infiltrated the alkali-burned corneas of the PBS-treated mice, and these cells expressed MMP-9 (Fig. 4). These data suggest that CD-ECM treatment inhibited the infiltration of neutrophils into the cornea, following an alkali burn, and also inhibited the production of neutrophil chemoattractants by corneal epithelial cells.

Chemokines have previously been implicated as crucial mediators of inflammatory cell recruitment (30). Therefore, the expression levels of neutrophil chemoattractants were compared between CD-ECM- and PBS-treated corneas. IL-8 expression levels were markedly reduced in the CD-ECM-treated corneas on day 3, as compared with the corneas from the PBS treatment group. IL- 8 is an important and representative $\mathrm{CXC}$-chemokine that is known for its potent ability to initiate chemotaxis and activate neutrophils (31). Corneal epithelial cells usually produce IL-8 in response to IL-1 $\beta$, TNF- $\alpha$, and IL- 6 stimulation, following an alkali burn (32). Upon stimulation by IL-8, neutrophils have been shown to express high levels of MMP-9 (33). The data of the present study suggests that CD-ECM treatment reduced the production of IL- 8 by the epithelial cells of alkali-burned corneas, and this resulted in the inhibition of the infiltration of MMP-9-expressing neutrophils.

In conclusion, the present study demonstrated that topical treatment with CD-ECM reduced corneal opacity, following an alkali burn. This treatment may facilitate reepithelialization and inhibit IL-8-mediated neutrophil infiltration. These results provide supporting evidence for the potential use of $\mathrm{CD}-\mathrm{ECM}$ as a novel therapeutic drug for the acute management of corneal chemical burns.

\section{Acknowledgements}

The present study was supported by a grant from the Korea Healthcare Technology R\&D Project, Ministry of Health and Welfare Affairs, Republic of Korea (no. HI12C0005).

\section{References}

1. Lee P, Wang CC and Adamis AP: Ocular neovascularization: an epidemiologic review. Surv Ophthalmol 43: 245-269, 1998.

2. Kuo IC: Corneal wound healing. Curr Opin Ophthalmol 15: $311-315,2004$. 
3. Adamis AP, Aiello LP and D'Amato RA: Angiogenesis and ophthalmic disease. Angiogenesis 3: 9-14, 1999.

4. Wagoner MD: Chemical injuries of the eye: current concepts in pathophysiology and therapy. Surv Ophthalmol 41: 275-313, 1997.

5. Brodovsky SC, McCarty CA, Snibson G, et al: Management of alkali burns : an 11-year retrospective review. Ophthalmology 107: 1829-1835, 2000

6. Ahmadi AJ and Jakobiec FA: Corneal wound healing: cytokines and extracellular matrix proteins. Int Ophthalmol Clin 42: 13-22, 2002.

7. Lu L, Reinach PS and Kao WW: Corneal epithelial wound healing. Exp Biol Med (Maywood) 226: 653-664, 2001

8. Ueno M, Lyons BL, Burzenski LM, et al: Accelerated wound healing of alkali-burned corneas in MRL mice is associated with a reduced inflammatory signature. Invest Ophthalmol Vis Sci 46 : 4097-4106, 2005.

9. Yao L, Li ZR, Su WR, et al: Role of mesenchymal stem cells on cornea wound healing induced by acute alkali burn. PLoS One 7: e30842, 2012.

10. Clements JL and Dana R: Inflammatory corneal neovascularization: etiopathogenesis. Semin Ophthalmol 26: 235-245, 2011.

11. Stortelers C, Kerkhoven R and Moolenaar WH: Multiple actions of lysophosphatidic acid on fibroblasts revealed by transcriptional profiling. BMC Genomics 9: 387, 2008.

12. Järveläinen H, Sainio A, Koulu M, Wight TN and Penttinen R: Extracellular matrix molecules: potential targets in pharmacotherapy. Pharmacol Rev 61: 198-223, 2009.

13. Chvapil M, Kronenthal L and Van Winkle W Jr.: Medical and surgical applications of collagen. Int Rev Connect Tissue Res 6 : $1-61,1973$

14. Stoop JW: Treatment of pressure sores in paraplegic patients with animal collagen. Paraplegia 8: 177-182, 1970.

15. Willoughby CE, Batterbury M and Kaye SB: Collagen corneal shields. Surv Ophthalmol 47: 174-182, 2002.

16. Friedberg ML, Pleyer U and Mondino BJ: Device drug delivery to the eye. Collagen shields, iontophoresis, and pumps. Ophthalmology 98: 725-732, 1991.

17. Mondino BJ: Collagen shields. Am J Ophthalmol 112: 587-590, 1991.

18. Altman RD and Moskowitz R: Intraarticular sodium hyaluronate (Hyalgan) in the treatment of patients with osteoarthritis of the knee: a randomized clinical trial. Hyalgan Study Group. J Rheumatol 25: 2203-2212, 1998

19. Mariggiò MA, Cassano A, Vinella A, et al: Enhancement of fibroblast proliferation, collagen biosynthesis and production of growth factors as a result of combining sodium hyaluronate and aminoacids. Int J Immunopathol Pharmacol 22: 485-492, 2009.
20. Jin CZ, Park SR, Choi BH, Park K and Min BH: In vivo cartilage tissue engineering using a cell-derived extracellular matrix scaffold. Artif Organs 31: 183-192, 2007.

21. Choi KH, Choi BH, Park SR, Kim BJ and Min BH: The chondrogenic differentiation of mesenchymal stem cells on an extracellular matrix scaffold derived from porcine chondrocytes. Biomaterials 31: 5355-5365, 2010.

22. Choi K-H, Song BR, Choi BH, Lee M, Park SR and Min B-H: Cartilage tissue engineering using chondrocyte-derived extracellular matrix scaffold suppressed vessel invasion during chondrogenesis of mesenchymal stem cells in vivo. Tissue Eng Regen 9: 43-50, 2012.

23. Ye HQ and Azar DT: Expression of gelatinases A and B, and TIMPs 1 and 2 during corneal wound healing. Invest Ophthalmol Vis Sc 39: 913-921, 1998.

24. Sharifipour F, Baradaran-Rafii A, Idani E, Zamani M and Jabbarpoor Bonyadi MH: Oxygen therapy for acute ocular chemical or thermal burns: a pilot study. Am J Ophthalmol 151: 823-828, 2011

25. Mostow EN, Haraway GD, Dalsing M, Hodde JP and King D OASIS Venus Ulcer Study Group: Effectiveness of an extracellular matrix graft (OASIS Wound Matrix) in the treatment of chronic leg ulcers: a randomized clinical trial. J Vasc Surg 41: 837-843, 2005.

26. Clements JL and Dana R: Inflammatory corneal neovascularization: etiopathogenesis. Semin Ophthalmol 26: 235-245, 2011.

27. Sotozono C, He J, Matsumoto Y, Kita M, Imanishi J and Kinoshita S: Cytokine expression in the alkali-burned cornea. Curr Eye Res 16: 670-676, 1997.

28. Kjeldsen L, Sengelov H, Lollike K, Nielsen MH and Borregaard N: Isolation and characterization of gelatinase granules from human neutrophils. Blood 83: 1640-1649, 1994.

29. Delclaux C, Delacourt C, D'Ortho MP, Boyer V, Lafuma C and Harf A: Role of gelatinase B and elastase in human polymorphonuclear neutrophil migration across basement membrane. Am J Respir Cell Mol Biol 14: 288-295, 1996.

30. Allen SJ, Crown SE and Handel TM: Chemokine: receptor structure, interactions, and antagonism. Annu Rev Immunol 25: 787-820, 2007

31. Harada A, Sekido N, Akahoshi T, Wada T, Mukaida N and Matsushima K: Essential involvement of interleukin-8 (IL-8) in acute inflammation. J Leukoc Biol 56: 559-564, 1994.

32. Xue ML, Willcox MD, Lloyd A, Wakefield D and Thakur A: Regulatory role of IL-1beta in the expression of IL-6 and IL-8 in human corneal epithelial cells during Pseudomonas aeruginosa colonization. Clin Experiment Ophthalmol 29: 171-174, 2001.

33. Masure S, Proost P, Van Damme J and Opdenakker G: Purification and identification of 91-kDa neutrophil gelatinase. Release by the activating peptide interleukin-8. Eur J Biochem 198: 391-398, 1991. 